\title{
Efectos del postconflicto: Una mirada crítica desde los derechos humanos
}

\author{
Effects of postconflict: \\ A critical look from human rights
}

\begin{abstract}
RESUMEN
Este articulo presenta una reflexión en torno al estado del arte que se construye acerca de las luchas por los derechos humanos de los colectivos que, en transiciones de conflictos internos, asumen procesos de transformacion politica y legal para posibilitar la paz. El constructo argumental tiene como epicentro el escenario de postconflicto que se vive Colombia.
\end{abstract}

PALABRAS CLAVE

Derechos humanos, Víctimas, Victimarios, Posconflicto, Paz.

\section{ABSTRACT}

The advance article presents the state of the art that is built around the struggles for the human rights of the groups that in transitions of internal conflicts assume processes of political and legal transformation to make peace possible. The argument construct has as its epicenter the postconflict scenario that Colombia is going through.

KEYWORDS

Human Rights, victims, Post-conflict, Peace.

\section{JOSÉ ARIEL PARRA VEGA}

Licenciado en Filosofía y Teólogo de la Universidad de San Buenaventura. Magíster en Docencia e Investigación Universitaria de la Universidad Sergio Arboleda. En este momento se desempeña como Coordinador de Investigaciones de la Escuela de Derecho de la Universidad Sergio Arboleda, Seccional Santa Marta. Se ha desempeñado como docente y pertenece al Grupo de Investigación GIJAM categorizado en A por Colciencias.

josearielparra@gmail.com

\section{CARLOS JULIO AGUDELO GÓMEZ}

Licenciado en Filosofía y Ciencias Religiosas de la Universidad Santo Tomás. Especialista en Gestión Educativa, de la Universidad de Pamplona. Especialista en Docencia e Investigación Universitaria. Magíster en Docencia e Investigación Universitaria de la Universidad Sergio Arboleda. Actualmente es profesor de tiempo completo, adjunto a la Gestión Académica de la Escuela de Derecho de la Universidad Sergio Arboleda, Santa Marta. carlos.agudelo@usa.edu.co

\section{RAÚL ALEJANDRO VIVIESCAS CABRERA}

Antropólogo de la Universidad del Magdalena. Estudiante de Especialización en Docencia e Investigación universitaria en la Universidad Sergio Arboleda, Sede Santa Marta. Actualmente se desempeña como Asistente de investigación en la Universidad Sergio Arboleda, Seccional Santa Marta. raulviviescasc@gmail.com 


\section{INTRODUCCIÓN}

Históricamente en Colombia se ha evidenciado el uso de la violencia por diversas causas en las que se pueden mencionar las diferencias partidistas, los conflictos armados, en las últimas décadas el narcotráfico y la delin- Muchos ciudadanos de toda condición socia cuencia organizada. En este contexto se han han sufrido las torturas, la desaparición forvisto involucradas las fuerzas militares, los zosa, la aniquilación de la familia, el desplagrupos guerrilleros, los carteles de las dro- zamiento, la violaciones en todas sus modagas, más recientemente las llamadas Bacrim lidades configurándose así los llamados de(Bandas Criminales). Todos estos actores se litos de lesa humanidad. han involucrado de manera directa o indirec- el mismo horror, pero sus acciones no son ta con la población civil, la cual finalmente ha significativas (Centro Nacional de Memoria sido la victima de situaciones que muchos de Histórica, 2013). Precisamente en el ar ellos no comprenden pero que han sido los lo 12 de la Constitución se dice que "Nadie que han puestos los muertos.

La Constitución del 91, tocó los temas anteriormente descritos, y dentro de lo legislado encontramos que: "Todas las personas nacen libres e iguales ante la ley, recibirán la misma protección y trato de las autoridades y gozarán de los mismos derechos, libertades y oportunidades sin ninguna discriminación por razones de sexo, raza, origen nacional, 0 familiar, lengua, religión, opinión, política o filosofía" (Gómez, 2014, p.10).

La protección a los ciudadanos ha tenido múltiples falencias y esto ha provocado masacres con algunas de ellas en la impunidad porque el ordenamiento jurídico se ha quedado corto para proceder con efectividad y eficiencia. La población civil termina por aceptar que los delitos se seguirán cometiendo y que los resultados, solo en algunos casos, llegan a la condena de los victimarios.
Uno de los casos más recurrentes que se ha venido presentando en Colombia por la situación de violencia que ha tenido que soportar por más de 50 años es la violencia en su máxima expresión. el mismo horror, pero sus acciones, no son Histórica, 2013). Precisamente en el artícuserá sometido a desaparición forzada, ni tortura, ni tratos o penas crueles, inhumanos o degradantes" (Gómez, 2014, p.10). Este artículo ha sido violado en su totalidad ya que la desaparición, las torturas y los tratos in dano del común se ha acostumbrado a esta absurda realidad que ya no es un hecho que lo trastorne.

En los últimos 20 años se han venido haciendo esfuerzos para lograr que la realidad violenta que vive Colombia llegue a su fin (Proceso de paz del Caguán 1998-2002; negociaciones y acuerdos con grupos paramilitares y Ley de Justicia y Paz, 2005). En el presente Gobierno, las conversaciones y la construcción de una justicia transicional permite ver la posibilidad de que los atropellos y la violencia generalizada lleguen a su fin, o por lo menos se entre a una tregua para poder construir una nueva sociedad. humanos son noticias cotidianas, y el ciuda-
Según estudios realizados por la CIDH (Comisión Interamericana de los Derechos Humanos de la OEA) en su informe: "Verdad, Justicia y Reparación", contextualiza la situación de Colombia y el gran impacto que sigue teniendo el conflicto armado interno. Dicen $y$ constatan que la guerra ha manejado todas las degradaciones que crean las múltiples formas de violencia, y su mayor preocupación es que se ha concentrado en sectores de mayor vulnerabilidad: afrodescendientes, raizales, palenqueros, niños, niñas, adolescentes, indígenas, mujeres, periodistas y comunicadores sociales y personas privadas de la libertad.

Esta investigación apunta a convertirse en un instrumento que facilite a las partes, pero especialmente a las víctimas y a los victimarios, para que conozcan sus derechos y los puedan exigir en el caso de que no se estén cumpliendo o se les estén vulnerando. Es claro que para que los diálogos de paz no queden en letra muerta y para que la paz sea sostenible en el tiempo se requiere que los involucrados en el conflicto tengan la percepción de que tanto los victimarios como e Gobierno estén cumpliendo los acuerdos y se pueda evidenciar el cambio de actitud de a población en general.

En este orden de ideas se hace pertinente esbozar la principal pregunta de investigación que guia este trabajo y que se formula de la siguiente manera: ¿Puede la vigilancia y las leyes de justicia, paz, reparación y no repetición, garantizar la sostenibilidad de los acuerdos alcanzados y que se pondrán en práctica en el postconflicto? Y como principales derivadas tenemos; ¿Cuáles han sido las experiencias en procesos de paz frente al respeto y cumplimiento de los derechos humanos en diferentes contextos? ¿Cuál es la percepción de las víctimas, los victimarios y la sociedad civil frente al cumplimiento de los acuerdos pactados en el proceso de paz para el postconflicto?

Los supuestos basicos sobre los cuales gira este artículo son:

1. Los problemas de violencia que ha vivido Colombia y los múltiples intentos que los Gobiernos han iniciado y que se han convertido en procesos fallidos, conducen a la sociedad colombiana a entrar en un ambiente de escepticismo puesto que no se quiere repetir un fracaso más. Por otro lado, las conversaciones que se han dado en los últimos seis años han despertado una luz de esperanza, y las estadísticas muestran que ha cambiado la percepción de la mayoría de los colombianos frente al presente proceso de paz que se está culminando, mostrando un apoyo generalizado (Angulo Amaya, Ortiz Riomalo, Pantoja Barrios, 2013). Esto permite pensar que el eventual acuerdo que pacten las partes en disputa esté fundamentado sobre bases sólidas y que tiene un nivel alto de confiabilidad para que el proceso de postconflicto, a partir del respeto a los derechos humanos adquiridos, puedan ser cumplidos por las partes y de esta manera construir de forma progresiva una nueva cultura ciudadana de paz $y$ armonía sostenible en Colombia. 
2. La normatividad y la justicia transicional que el Gobierno ha implementado para el postconflicto muestra algunas dificultades de interpretación, lo cual puede conducir a que después de la firma de los acuerdos de paz las partes le hagan una lectura sesgada a la ley interpretándola a su favor y se presenten dificultades legales frente a las conductas desarrolladas por el Gobierno o por los desmovilizados. En este escenario el postconflicto entraría en una laguna jurídica y en un laberinto donde las partes alegarían las razones que los asisten y se pondría en peligro fundamentalmente la paz y los derechos humanos que las partes se comprometieron a respetar bajo cualquier circunstancia.

1. Las luchas por los derechos como luchas por la paz

Las libertades y los derechos han tenido en la historia un enfasis especial en las discusiones con respecto a la presencia de estos en la vida del Estado. Los ciudadanos se han visto abocados a reclamar y los gobernantes a definir y aclarar cuáles son los verdaderos alcances de las libertades y los derechos que les asisten a los ciudadanos en cada momento histórico. En su libro Los Derechos Fundamentales Mauricio Fioravanti (2009), nos dice sobre el tema:

El Estado medieval tiene grandes diferencias con respecto al Estado moderno, (...) no se debe cometer el error de buscar Derecho en la Edad Media utilizando las categorías del Derecho moderno; si se hace de esta manera fácilmente se concluye con la ausencia de Derecho en el Medioevo, precisamente porque así no se busca de ningún modo el derecho propio y específico de la Edad Media sino el mismo Derecho moderno, es decir, algo que se ha afirmado más tardíamente. (2009, p.27)

Cada momento histórico ha mostrado los mecanismos que se utilizaron para garantizar los derechos y las libertades, pero estos mecanismos ya no son válidos en nuestro contexto histórico. Repetimos, sería un error sumergirnos en un contexto histórico ajen al nuestro, puesto que se caería en una interpretación carente de actualidad y de veracidad. En la Edad Media raramente se hacían valer los derechos y las libertades, y esto $\mathrm{n}$

significa que en la Edad Modern continuado con esa costumbre; en esta edad los individuos dependían de su origen y de su lugar al cual pertenecieran, de tal maner que los derechos y las libertades eran patrimonio del feudo y los individuos terminaban gozando de estos derechos y libertades solo si estaban adscritos a una ciudad o a una ldea, etc. En contraste con esto Fioravan argumenta:

(...) todo esto es incompatible con la concepción moderna de la libertad como libre expresión de la voluntad, como libertad positiva. A esta dimensión de libertad, irrenunciable en el derecho moderno, se opone de modo irreconciliable el mundo medieval, que, en el mismo momento en que confía los derechos y las libertades a la fuerza del orden natural de las cosas históricamente fundado, impide a los hombres disfrutar de la esencial libertad de querer un orden diferente, es la falta de esta libertad, que en su raíz es la progenitora de las libertades políticas, las positivas lo que nos hace sentir -a nosotros los modernos- la edad media como algo lejano. (2009, p.31)

Encontramos que existen profundas diferencias en los dos contextos históricos de los que se habla, es posible que en la Edad Media los mecanismos para hacer valer los derechos y las libertades sean vistas en su momento como válidas puesto que el orden natural así lo entendía; en la Edad Moderna con un tinte más positivista, el individuo puede disfruta de los derechos y las libertades puesto que son asumidos por el individuo y defendidos por el Estado. Continuando el camino que nos muestra la historia de la aparición de los Derechos y las libertades, se encuentra que la discusión ha sido permanente por la interpretación que se le han dado a los derechos en el momento en que el Estado debe ponerlos en la práctica en una sociedad concreta y unos ciudadanos concretos.

En el siglo XVIII y en el XIX los Estados Europeos y el Estado americano lograron decantar la fuerza y validez que las libertades y los Derechos tenían para los individuos, es as que:

Derecho Constitucional europeo contemporáneo reconoce a los individuos un patrimonio de derechos, originario, independiente y protegido frente a la ley. En este punto se separa de la concepción revolucionaria francesa y se aproxima a la tradición constitucional norteamericana. (Zagrebelsky, 2011, p.54)

Para la concepción americana los derechos son anteriores tanto a la Constitución, como al Gobierno (al Estado). Según la famosa argumentación circular del federalista de ascendencia lockeana, las Cartas Constitucionales eran el acto mediante el cual el pueblo soberano delegaba libremente en los gobernantes. El poder de estos se basaba en esa delegación, y naturalmente, debía permanecer dentro de los límites marcados por ella, más allá de los cuales se produciría la absoluta nulidad jurídica de sus actos (Zagrebelsky, 2011, p.55)

Es evidente que los teóricos han tenido diversas y divergentes posiciones sobre las libertades y los derechos humanos. Lo que es claro es la existencia de estos por derecho natural, para unos y por derecho positivo para otros. Esto quiere decir que los gobernantes velarán por el respeto de los derechos humanos dependiendo la dirección teórica que consideren la mejor y aquí es donde se pueden cometer arbitrariedades relativas la tendencia academicista y política en la cual hayan sido formados.

Con referencia al plano internacional debemos destacar de manera importante el conflicto y camino hacia la paz que realizó Irlanda del Norte, donde según informes y escritos que han hecho seguimiento a las conversaciones que llevan el Gobierno y las FARC (Fuerzas Armadas Revolucionarias de 
Colombia). El grupo guerrillero ha puesto en la mesa de negociación el modelo de Irlanda conocido como Acuerdo de Viernes Santo que se dio el día 10 de abril de 1998. Citaremos así de manera precisa el informe de Jorge Alberto Mantilla:

El conflicto de Irlanda del Norte tiene su origen en una serie de incompatibilidades históricas relacionadas con la colonización británica de la isla durante más de cuatro siglos y a las estructuras de poder político, social y económico que de esta derivaron. Lo fundamental que generó el conflicto era una disputa etno-religiosa entre católicos nacionalistas y protestantes unionistas, estas variables están sustentadas en una estructura de poder territorial y político que reprodujo una violación estructural de tipo económico y cultural contra los Irlandeses católicos. (2014)

Entonces según lo expuesto por Mantilla (2014) el proceso duró más de una década, desde 1987 hasta 1998. Este hecho hace que lo vivido en Irlanda del Norte constituya e caso más importante internacional y modelo a seguir para la resolución de conflictos armados. Varios factores y personajes importantes formaron un bloque para que se diera la paz en Irlanda. Estos fueron: un favorable ambiente, el cansancio de los protagonistas o bloque armado, necesidad del gremio económico de lograr la paz y el apoyo del nuevo presidente de Estados Unidos (Bill Clinton), pero lo que primó y favoreció al acuerdo de paz fue el movimiento que creó la sociedad civil que se convirtió en un mandato cívico por la paz.

De acuerdo con Mantilla (2014) el Acuerdo de Viernes Santo, en uno de sus apartes principales menciona de manera especial e reconocimiento del sufrimiento y dolor de las víctimas del conflicto armado. Por esta razón crearon ambientes de reconciliación realizando convocatorias públicas que dieron origen a las estrategias fundamentales para la reparación de las víctimas, dejando claro que el Estado se comprometía a: a) reconocimiento por ayuda material; b) reconocimiento por proyectos conmemorativos $y$ c) se creó la figura del defensor cuya labor debía ser la de consejero y orientador a las víctimas.

En 1990 crean el CRC (Consejo de Relaciones Comunitarias); su función era: La elaboración de proyectos con personas que estuvieran interesadas en afrontar el conflicto de manera productiva. La labor realizada por el CRC fue vital e importante para que se diera la reconciliación a nivel local, dejando as las bases claves para que el acuerdo de 1998 diera resultados positivos. Otro organismo de gran importancia para la resolución de este conflicto modelo fue el programa Peace, apoyado y financiado por la Comisión Europea, encargado en su primera etapa de "promover la reconciliación a través del impulso y desarrollo económico y el empleo, los centros urbanos y de las zonas rurales, el desarrollo de la comparación en las fronteras y la ampliación de la inclusión social" (Hamber \& Gráinne, 2005, p.5)
Si mencionamos en este proceso el desarme, que como en todo proceso de paz debe ser el eje principal, en el acuerdo de Irlanda del Norte, este tema puso un traspié ya que el IRA (Ejército Republicano Irlandés - IRIS Republican Army), protagonista y actor principal del conflicto, se negó a realizar una entrega tradicional de las armas ya que para ellos se convertía en una figura de rendición o ser vencidos

Con respecto a la situación que vivió Nicaragua, con sus efectos políticos y consecuencias sociales del conflicto armado abordamos el análisis realizado por Berenice Cortés Rincón (2014) quien, de manera especial relata el principio del conflicto, desarrollo, postconflicto, paso a paso y análisis de la situación actual que vive Nicaragua después 25 años del "fin del conflicto", firmas de paz y acuerdos. Y se señala fin del conflicto entre comillas ya que esta autora nos narra que en la actualidad se vive una experiencia peor que la misma guerra, llevando al país a su capital Managua principalmente, a los indices más altos de hambre, dolor y extrema pobreza.

Se evidencia así que la conclusión de la guera, firmas de tratados o pactos no implica realmente la terminación del conflicto. En Nicaragua el $46,2 \%$ de los hogares viven en situación de pobreza y el $14,9 \%$ en extrema pobreza; es el segundo país más pobre de América.

Centroamérica es considerada hoy la región más violenta del mundo. Honduras tiene el récord mundial en homicidios, Guatemala el de feminicidios y en El Salvador hay más muertos por asesinatos que los que morían en años de la guerra. Los índices de violencia sexual y los de violencia de género son escalofriantes (Cortés, 2014, p.81).

Aparte, Hernández Pérez (2012) relata la vioencia de El Salvador en los años 80 durante su proceso de postconflicto, siendo de carácter político que generó la guerra civil que el Estado enfrentó contra la Guerrilla del FMLN (Frente Farabundo Martí de Liberación Nacional), apoyado por la intervención de Estados Unidos, en cabeza del presidente Reagan que mostró el interés de combatir la subversión comunitaria que era apoyada por Cuba, Nicaragua y la Unión Soviética.

Hernández (2012) nos muestra la violencia desarrollada en medio de un país hundido por la pobreza y la desigualdad que deja grandes marcas e índices por los crímenes cometidos por las mismas fuerzas armadas del Estado contra la población civil, la negación del Gobierno a dichos crímenes, existiendo evidencia plena. Se recopila en este artículo la estadística que para los años 1980 y 1981 ocurrieron 21.000 asesinatos de civiles en manos de las fuerzas públicas del Gobierno.

En conclusión, se establecieron avances más en paz que en democracia, en desmilitarización que en vigencia de los derechos humanos y justicia y en reformas constitucionales más que en cuestiones económicas y sociales (Hernández Pérez, 2012, p.3). 
En cuanto a la revisión académica referente sición y posterior postconflicto, están comal conflicto que tuvo lugar en Argentina y Chi- prometidos y bajo vigilancia por organismos le referenciamos a Elin Skaar (2003), quien internacionales para que de una u otra forma aborda la temática respondiendo a una pre- hagan valer los derechos humanos vulneragunta clave: ¿Cómo ocuparse de los agentes dos y creen una estrategia óptima para resde violaciones masivas y sistemáticas de los tablecer el daño causado y poder construi

derechos humanos después de la transición políticas públicas que lleven al Estado a la no del autoritarismo a la democracia?

Pareciera ser ante esta pregunta del autor que después de la etapa de transición, los Gobiernos mediante un silencio y falta de actuación pretendían que aquellos delitos $y$ violaciones cometidos durante la etapa de conflicto se fueran olvidando $y$, pareciera que en algunos países esto funcionó porque de quince paises que vivieron transiciones

la democracia, únicamente Argentina tuvo un juicio a altos jefes de su cúpula militar imponiéndoles importantes penas carcelarias. Después de una década se sigue buscando contra quién o quiénes puede el Estado enjuiciar a aquellos militares por el secuestro de un centenar de niños.

Elin Skaar (2003) también comenta que quiere llenar un vacío con su investigación acerca de que no se deja en claro el papel de la Rama Judicial en las decisiones tomadas durante los acuerdos de paz y posteriores actuaciones en el llamado postconflicto. Señala que estos acuerdos siempre van acompañados por reformas constitucionales parciales para darle a algunos el poder e independencia judicial frente al Poder Ejecutivo.

Estados como Argentina y Chile acá analizados, y aquellos que entran a régimen de tranrepetición de estas violaciones.

Alrededor de 15 países han atravesado transiciones de regímenes autoritarios a democráticos y a finales de los años 70 tuvieron brutales dictaduras militares en las que se cometieron las peores violaciones a los derechos humanos contra la misma población civil. Después de 7 años de la etapa de transición a la democracia, en Argentina el Poder Ejecutivo apoyaba una política de "perdón y olvido" y controlaba a los jueces para que no llegaran al final de las investigaciones contra militares y miembros de las fuerzas armadas. Entre tanto, movimientos locales por la defensa de los derechos humanos como las madres de la Plaza de Mayo ejercían presión al Gobierno poniéndolo en observación a nivel internacional.

Con referente a lo que acá se señala de la situación chilena donde la dictadura duró 17 años, su terminación no ocurrió como la de Argentina, que fue por un colapso. En Chile se dio por medio de conversaciones, negoles que llevaron a que el dictador Pinoche perdiera las elecciones que él mismo promovió. La dictadura dejó para Chile, legados imborrables. Una Constitución impuesta 1980 que garantizaba el poder de las fuerzas ciaciones y acuerdos entre las clases socia- rmadas y militares con primacía absoluta y aplastante, caracterizada por la ausencia de juicios. Por esta razón, quedaron en la impunidad todos los delitos y violaciones a los derechos humanos. Ambos países (Argentina y Chile) a diferencia de los países de Centroamérica (Nicaragua y El Salvador) han tenido una lucha constante por restablecer derechos y recuperar social y económicamente a sus naciones.

Ahora, al tornar la mirada para ver lo que sucede con los derechos humanos en Colombia, se puede interpretar que son puestos en práctica atendiendo a una visión incluyente de varias teorías que hay sobre el tema; una referencia clara de la posición subjetivista de los derechos la encontramos en la Constitución Nacional de Colombia en el artículo 5 que a la letra dice: "El Estado reconoce, sin discriminación alguna, la primacía de los derechos inalienables de la persona (sujeto) y ampara a la familia como institución básica de la sociedad".

Con respecto a esto, Zagrebelsky (2011) en su libro El derecho dúctil expone que en Europa y especialmente en la Francia del siglo XVIII y en adelante, los derechos humanos eran una concesión de la ley a la persona. Esto quiere decir que se privilegiaba la ley los derechos humanos, que finalmente dependían del legislador o del gobernante. A contrario, en la Constitución americana los derechos están por encima de la ley en cuanto que el individuo es el que ha delegado a gobernante para que vele por los derechos que se encuentran de la ley; aquí priman los derechos sobre la ley por lo tanto:
Los derechos, por cuanto patrimonio subjetivo independiente, que constituía a los individuos en sujetos activos originarios $y$ soberanos y de este modo hacían posible el acto de delegación constitucional fundando el Government y, en él, el Poder Legislativo. La ley, cabe decir, derivaba de los derechos, justamente lo contrario de lo que sucedía en Francia donde eran los derechos los que derivaban de la ley. Aquí la soberanía de la ley; allí, la soberanía de los derechos. (p.55)

2. Análisis del caso colombiano: el postconflicto como superación histórica de la vio-

Es evidente que los derechos humanos se convirtieron para el Estado colombiano en un renglón importante de su ordenamiento jurídico por el compromiso que adquirió al firmar algunos acuerdos internacionales que ponían en tela de juicio el manejo que los Gobiernos les estaban dando a los conciudadanos. Una referencia que ilustra esta realidad la encontramos en el historiador Melo González, el cual plantea que:

La Constitución de 1991 incorporó, además, al inventario de derechos humanos varios derechos económicos y sociales (empleo, vivienda, salud, cultura, etc.) e incluso convirtió en derechos de los ciudadanos algunos de los objetivos básicos del orden constitucional, como la paz. De este modo, la Constitución no solamente señaló los derechos exigibles del Estado, sino que definió como derechos muchos temas 
que normalmente hacen parte de la con- también puede ser el comienzo de una nuetroversia política. Al hacerlo, tendió a des- va etapa de violencia. Coherente con esta politizar la búsqueda de metas sociales y a preocupación, distintos autores han abordacrear un ambiente en el que el desarrollo do esta problemática y han realizado distineconómico y social no se logra a través de tas propuestas entre las cuales destacamos la política y la participación democrática, el trabajo de María Victoria Llorente, Sergio sino mediante demandas en los tribuna- Guarín y Ángela Rivas (2014), quienes recoles. (Melo González, 2002)

En el contexto actual vuelven a tomar fuerza los derechos humanos en cuanto que es un requisito para que los acuerdos que se firmen para la búsqueda de la terminación del conflicto armado puedan tener credibilidad por parte de las víctimas, de los victimarios y de la sociedad civil la cual ve con escepticismo la firma de la paz.

Para que el Gobierno y el grupo insurgente garanticen los derechos humanos, será fundamental construir una cultura del respeto $y$ de la reconciliación; dentro de esta construcción los canales de información sobre la vigilancia y el cumplimiento por las partes de los derechos humanos debe ser implementada con veedores de alta credibilidad. Esto ayudará a que si se presenta alguna dificultad, haya alertas tempranas para poder superar cualquier dificultad; el conocimiento de los derechos humanos se deberá convertir en una tarea educativa fundamentada en un plan nacional que debe ser promovido especialmente por el Gobierno.

Como ya se ha puesto en evidencia, se acerca un momento histórico clave para Colombia como ya varios autores citados han expuesto, así como el conflicto puede llegar a su fin, nocen el momento crucial por el cual pasa país y además señalan -citando a Jaramillo (14 de mayo 2013)-que el postconflicto que está por llegar será la oportunidad en la que realmente se decidirá el futuro de todo este proceso.

Así mismo señalan varios elementos que se deben tener en consideración dentro de esta etapa: El primero de ellos -tal vez el más importante- es reconocer que el conflicto colombiano va más allá de las problemáticas más visibles (narcotráfico, guerrillas...), que si bien es un punto importante, no deja entrever la raíz histórica de este fenómeno que refiere a un problema de tipo estructura.

Seguidamente, y apoyados en el constructo argumental de Robinson (2013) y Robinson \& Acemoglu (2012), sugieren la necesidad de una trasformación a las estructuras de pode predispuestas desde la época colonial que configuró la institucionalidad del país a largo plazo, institucionalidad de depredación que diagnostican como "caldo de cultivo perfecto para la corrupción, la violencia y la ilegalidad". Proponen una transformación en la institucionalidad colombiana que, coherente con la problemática histórica, revierta las problemáticas de gestión y manejo territoria creando una institucionalidad inclusiva.
Sin lugar a dudas, lo más destacable de la reflexión anterior es la de preguntarnos por el cambio que se acerca, para que nuestra noción de paz no quede atada a un discurso común, sino que reconozca el contexto geohistórico del conflicto colombiano que plantee un debate abierto y constructivo. En su análisis nos recuerda que el postconflicto es una etapa de cambios, y como tal rae incertidumbres; tenemos que darnos la oportunidad de arriesgarnos -proponiendo, creando, investigando...- para poder erigir un mejor futuro (Kotter, 2012).

Ia misma línea encontramos el trabajo de Borja Paladini (2014) quien investiga si Colombia dispone de un sistema complejo e interdependiente de instituciones, recursos, valores, estrategias y habilidades para impulsar una paz sostenible -para la cual hace una interesante analogía con las fuerzas armadas-. Esta investigación la resuelve enseñando diferentes ideas que aportan a este debate: La primera de ellas nos habla desde el concepto planteado por Ryan Jordan (2012) cual nos habla de una "infraestructura de paz" que se debe entender como un entramado de sistemas, recursos, instituciones, valores y habilidades sostenidas por un Gobierno, la sociedad civil con las diferentes comunidades, quienes a través del diálogo y consulta previenen conflictos, y a su vez, posibilitan un arbitraje pacífico.

En síntesis, Borja Paladini (2014), con esta orientación introduce a la importancia de la responsabilidad que tienen todos los estamentos del Estado (instituciones, hombres, De forma última, en esta revisión se desta-

mujeres...) en la construcción de la paz, que "como un proceso endógeno de trasformación y de innovación" solo será sostenible y resiliente si brota de las personas, las víctimas, los hombres, las mujeres, los victimarios, la población joven, las instituciones del país, y-como ya se mencionó- a todas las personas.

En coherencia con su tesis principal, este autor plantea unas ideas acerca de las características que debería tener una infraestructura para la paz en Colombia, proyectando combinar lo acordado -eventualmente- con as diferentes iniciativas y experiencias institucionales y comunitarias, de las cuales se destacan las siguientes:

- Desde lo nacional: Un sistema de gobernanza de la paz, que permita coordinar y asistir una red institucional que sirva de apoyo a la implementación de los futuros acuerdos, así como la creación de instituciones de apoyo para este fin.

- Desde lo regional: Con la apertura de espacios que propicien acercamientos entre lo nacional y lo local, abriendo un campo de acción idóneo para contextualizar en lo local las diferentes políticas y programas nacionales que incluyan un eventual acuerdo

- Desde lo local: A través de los fortalecimientos de los canales de participación ciudadana; por ejemplo, comités locales de paz que permitan un diálogo plural que nutra y enriquezca la implementación de los acuerdos de paz.

REVISTA VIS IURIS I No. 4, Vol. 2181 - 95 I Juilo-Diciembre, 2015 I Universidad Sergio Atboledal I Escuela de Derecho I Seccional Santa Marta, Sede Centro 
ca el análisis realizado por Benavides (2014), sables de grandes violaciones de derechos quien aborda una temática controversial. La humanos. Entonces -de acuerdo al segundo justicia en el periodo de transición que se punto "justicia e impunidad"- es vital que se avecina es un tema obligado para esta inves- respalde la investigación y el juzgamiento de tigación, debido a que esta justicia será la los autores de las graves violaciones de los que dotará al Gobierno del mecanismo para derechos humanos, logrando que la postura hacer frente al proceso de paz, a través de lo de las víctimas sea más que retórica. que se ha denominado marco jurídico para la paz.

\section{CONCLUSIONES}

En relación con lo anterior, Benavides (2014) Para cerrar el artículo es importante menrealiza un importante aporte aclarando el cionar las nuevas expectativas de diferentes concepto de justicia transicional -entendida comunidades históricamente afectadas por desde el Gobierno- además de introducir en el conflicto armado en Colombia frente a la problemática de justicia e impunidad que postconflicto (caso particular de varios de los toca acápites importantes -en esta investi- habitantes de Chibolo y sabanas de San Ángación- como los derechos humanos, entre gel y, en especial el grupo étnico Ette Ennaka, otros.

\section{en el Magdalena).}

El primero de estos conceptos -Justicia tran- Vemos cómo se evidencia una transición de sicional- provir tre el eventual acuerdo de paz y la fase de construcción de la paz, lo que se denomina etapa de transición. El autor destaca que en la primera fase de este proceso se sientan las bases y se plantean los caminos posibles para la construcción de la paz, en aras de cumplir un objetivo principal, el fortalecimiento del Estado de Derecho que se traduce en el fortalecimiento de un orden o unas reglas d juego.

De ahí que la importancia de la no impunidad, añadiendo que esta se mide por el grado de satisfacción de los derechos de las víctimas. Aunque también aclara que uno de los derechos importantes de las víctimas es que se investigue y se sancione a los responestos pueblos que han pasado del autorreconocimiento a un sentido de pertenencia social reivindicando su igualdad de derechos, $y$ en algunos casos -como el del pueblo Ette Ennaka- sus diferencias como enfoque de acción frente a su realidad. En otras palabras, el postconflicto lo asumen como un reto local, dentro del cual se plantean estrategias de perdón, recuperación del tejido social reconciliación con sus victimarios, proceso que reconocen como independiente al proceso realizado por parte del nivel nacional.

Por otra parte, estas comunidades que han participado abiertamente en el proceso de reparación colectiva en un diálogo de cohesión social con el Estado, son evidencia de cómo los sectores históricamente excluidos tienen la necesidad de reconocimiento de Sin embargo el proceso de acercamiento de sus derechos reivindicando su igualdad y diferenciación.

la reparación colectiva mostró una serie de limitaciones, a pesar de que las comunidades se muestran prestas $y$ atentas a participar de Aclarando lo anterior, el concepto de repara- estas actividades con el objetivo de romper ción colectiva aparece definido como el be- el aislamiento que han sufrido por cuenta de neficio que se confiere a un sujeto colectivo, la geografía, la violencia y el Estado. con la finalidad de reparar el daño causado como consecuencia de la violación de los De- Todas estas características permiten reconorechos Humanos que las leyes internaciona- cer la necesidad de un cambio estructural les vigilan (Rombouts, 2004). Los sujetos de que permita que estas iniciativas sociales y reparación colectiva es un término introduci- políticas no sean excluidas, dando cuenta de do por Rosefeld (2010) quien los define como la necesidad de una transformación de la golos beneficiarios de la reparación colectiva y bernabilidad, que permita el reconocimiento señala que pueden ser grupos reconocidos de las necesidades de estos grupos histórilegalmente y/o un conjunto de individuos.

El mismo autor identifica también el daño colectivo, como el que es causado por la violación de las leyes internacionales, sugiriendo que la reparación colectiva debe diferenciarse del deber de los Estados por garantizar los derechos humanos de sus ciudadanos, definidos también por la CNRR como:

Un derecho fundamental del cual son titulares los grupos, pueblos, u organizaciones sociales y políticas que hayan sido afectadas por el daño ocasionado por la violación de los derechos colectivos, la violación grave y manifiesta de los derechos individuales de los miembros de los colectivos, o el impacto colectivo de la violación de derechos individuales. La reparación colectiva comprende medidas $d e$ restitución, compensación, rehabilitación satisfacción y garantías de no repetición, en los componentes político, material $y$ simbólico...". (2010) camente marginados, propiciando espacios de solidaridad e inclusión y no solo reconociendo al otro, en función de políticas económicas, eficiencias productivas o parámetros de estatutos internacionales, corrigiendo un error estructural histórico.

\section{REFERENCIAS}

Angulo Amaya, M. C., Ortiz Riomalo, A. M. y Pantoja Barrios, S. (2013). Análisis de las percepciones de los colombianos sobre el proceso de paz y el postconflicto desde una perspectiva de género. En Revista Colombia Internacional, Universidad de los Andes. Disponible en: http://colombiainternacional.uniandes.edu.co/view.php/9165/index. php?id=9165

Benavides Vanegas, F. S. (2014). Cómo encarar la justicia en el periodo de transición. En Por la Paz. Colombia en el postconflicto violento, 20. Abril 2014. Institut Català Internacional per la Pau (ICIP). 
Comisión Interamericana de los Derechos Humanos (2013). Verdad, justicia y reparación: Cuarto informe sobre la situación de derechos humanos en Colombia. OEA/Ser.L/V/ II. Doc. 49/13.

Cortés Rincón, B. (2014). El proceso de paz de Nicaragua, efectos politicos y consecuencias sociales del conflicto armado (Tesis de maestría). Universidad Católica de Colombia Facultad de Derecho, Bogotá D.C., Colombia.

Fioravanti, M. (2009). Los Derechos Fundamentales. Apuntes de historia de las Constituciones.

Gómez Sierra, F. (2014). Constitución Política de Colombia. Básica. Bogotá, Colombia: Ed Leyer.

Hernández Pérez, T. (2012). El papel de la democracia en sociedades postconflicto, situación del conflicto armado interno: El caso del Salvador y Colombia.

Informe General Centro Nacional de Memoria Histórica (2013). iBasta Ya! Colombia: Memorias de guerra y dignidad.

Jaramillo, S. (14 de mayo de 2013). Transición en Colombia ante el proceso de paz y la justicia. El Tiempo.

Kotter, J. (2012). Leading Change. Boston: Harvard Business School Press.

Ley 975. Por la cual se dictan disposiciones para la reincorporación de miembros de gru- pos armados organizados al margen de la ley que contribuyan de manera efectiva a la consecución de la paz nacional y se dictan otras disposiciones para acuerdos humanitarios. Diario Oficial No. 45.980 de 25 de julio de 2005

Mantilla, J. A. (2014). El Acuerdo de Viernes Santo o la paz como nuevo diseño institucional compartido: Lecciones del proceso de paz en Irlanda del Norte. En: Políticas Públicas, (5), julio 2014. Universidad Nacional de Colombia.

Paladini Adell, B. (2014). Infraestructura de paz: hacia un sistema de apoyo a la construcción de paz en Colombia. En: Por la Paz. Co lombia en el postconflicto violento, 20, abri 2014. Institut Català Internacional per la Pau (ICIP).

Robinson, J. \& Acemoglu, D. (2012). Por que fracasan los países: los orígenes del poder, la prosperidad y la pobreza. Bogotá: Planeta.

Robinson, J. (2013). Colombia: Another 100 Years of Solitude? In Current History, 43-48.

Rombouts, H. (2004). Victims Organization and the Political or Reparation: A Case of Study on Rwanda. United States of America: Ant-werp-Oxford.

Ryan, J. (2012). Infrastructures for Peace as a Path to Resilient Societies. An Instituciona Perspective. En: Journal of Peacebuilding \& Development, 7(3), 15
Skaar, E. (2003). Independencia judicial y política de derechos humanos en Argentina $y$ Chile. Bogotá, Colombia: Ed. ILSA.

lombia en el postconflicto violento, 20 , abril 2014. Institut Català Internacional per la Pau

Victoria Llorente, M., Guarín, S. \& Rivas, Á. Zagrebelsky, G. (2011). El derecho dúctil. Ley, 2014). La construcción de la paz en Colom- derechos, justicia.

bia y el reto del cambio. En: Por la Paz. Co- 